\title{
BIM AND CLOUD PLATFORMS FOR FACILITY MANAGEMENT OF ROMAN TEMPLE "HADRIANEUM": CHAMBER OF COMMERCE IN ROME - DIGITALIZATION AS A SOLUTION FOR HISTORICAL HERITAGE MANAGEMENT
}

\author{
DAMIANO DI CIACCIO, EDOARDO MARODER, SARA AMBROSIO \& FRANCESCO PACCANICCIO \\ Rilo Digital Planning S.r.l., Italy
}

\begin{abstract}
Facility and maintenance management can be made much more efficient and cost-effective by using a digital twin of the building. In this article we present the GIM protocol which constitutes an innovative approach for maintenance management through the creation of a digital twin interconnected to the existing building. It uses a BIM model that is constantly updated on the characteristics and status of the building. To show the interest of our proposal we present a case study concerning the digitization process of the Hadrianeum in Rome, one of the most representative buildings in the city and headquarter of the Rome Chamber of Commerce.
\end{abstract}

Keywords: BIM, facility and maintenance management, cloud computing, building digital twin.

\section{INTRODUCTION}

The diffusion of the BIM methodology is profoundly transforming the different phases of the building process. This transformation involves professional skills, equipment and organizational structure.

On the other hand, the digitalization process that has involved many other sectors, in the construction sector is still very backward and there is a widespread lack in terms of archiving and consultation of documents, monitoring of structures and systems, of collections. of data concerning the history of the building and the management of essential services.

This paper intends to present an innovative management approach, characterized by ease of use, the intuitiveness of the graphic interface and the immediacy of the results, expressed in schematic form through visualization dashboards. The paper takes up the concepts already exposed in [1] providing further details.

We will initially introduce a general framework of the problems involved, then the proposed solution and the results that can be achieved will be illustrated with a case study concerning the digitalization process of the Hadrianeum in Rome, one of the most representative buildings in the city and headquarter of the Rome Chamber of Commerce. In the conclusions we will outline some possible developments.

\section{THE DIGITAL TRANSITION}

Senaatti's experience in the digital management of Finnish public assets [2] has demonstrated the usefulness of using parameterized digital projects. A more limited experience is the English one which, albeit on a smaller scale, has also led to the definition of a standard dedicated to the management of assets and their maintenance, the COBie [3]. These experiences have clearly shown that through digitization it is possible to dynamically monitor all the technical and economic characteristics of the project.

The underlying problem for such techniques is interoperability. IFC (Industry Foundation Classes) is an open data format, created to facilitate interoperability between the various 
operators. This standard aims to allow the sharing of an information model without loss or distortion of data or information. The definition of IFC is aimed at the coding of all building information, throughout the life cycle, from the feasibility analysis to its construction and maintenance, passing through the various design and planning phases

However, digital technologies are emerging and will certainly have a great impact in the construction and management of a building. The advent of 5G and the increasingly widespread use of sensors will allow for constant monitoring of the building in terms of component movement, temperature, ventilation, air quality, etc. Such a large and continuous collection of building data will allow the application of automatic collection and surveillance systems based on artificial intelligence. The advantage of these advanced procedures is in the evident saving, in terms of time and data consistency, compared to the use of current BIMdatabases which, although dynamic, require a substantial intervention of the operator in the insertion of the measures or in the extrapolation of the data.

On the other hand, the evolution towards an interconnected digital system, in a context such as the European one, where most of the building stock is existing or historical, and the companies are mainly small and not very innovative, requires quite a long time

\section{OUR PROPOSAL}

Our proposal consists of an Integrated Maintenance Management (GIM). This provides a protocol that includes an application methodology focused on the digitalization of architectural-engineering elements, aimed at their management. In a first phase, this procedure is based on the verification of the real state of the construction in question and consequent reproduction through digital BIM modeling. The final result is the creation of a digital twin of the property, containing all the technical, construction and documentary information relating to its current state, which populate a database in a dynamic and continuously updated BIM environment.

Once the computer database has been created, the next step is to use and enhance the digital information available, through the use of customized methodologies, which allow the traditional ordinary and extraordinary maintenance process to be managed electronically. The tools defined in this way lead to a continuous collaboration and integration of the computer data on the building with the dynamic procedures of the management area, through the use of external databases connected in a bidirectional network with the current state of the building.

The first fundamental step for the creation of the protocol is given by the evaluation of what are the real expectations and needs of the client compared with the starting information and technical material available. Starting from these considerations, a preliminary overview of the actual development of the protocol is obtained in terms of times, needs, costs and benefits. By balancing these four fundamental parameters to summarize the correct relationship from time to time, the scope of work and the procedures necessary to meet the highlighted needs are clearly defined.

Once any gaps found in terms of data and/or documentation have been filled with the tools available, we proceed to integrate what is necessary for the development of the protocol according to the established purposes, through various types of activities (non-invasive surveys analysis, laser scanner, thermographic surveys, due diligence, etc.). 


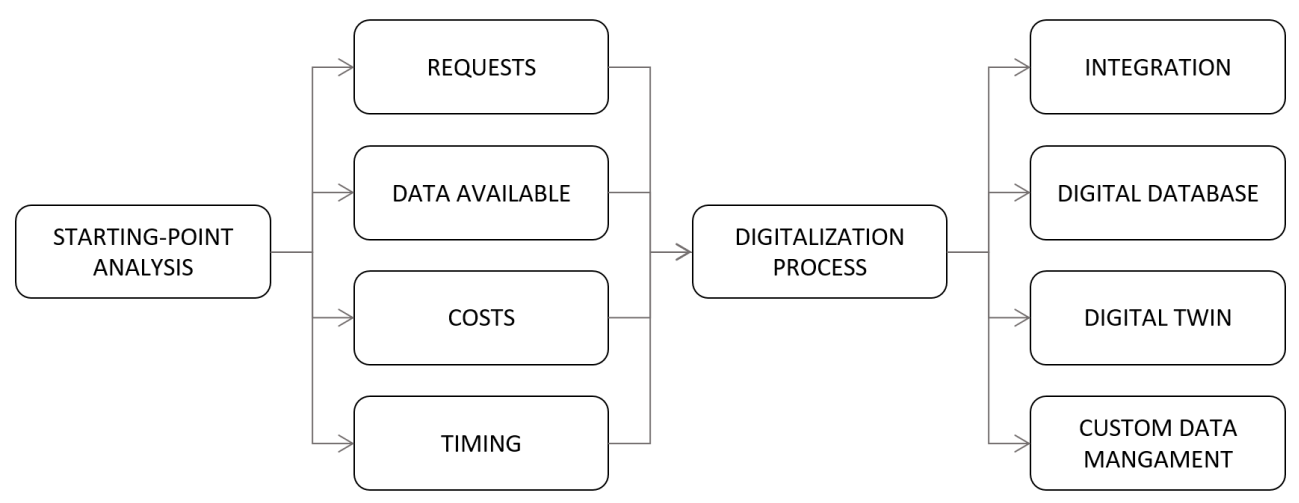

Figure 1: Functional diagram: Analytical phase.

\section{CREATION AND MANAGEMENT OF THE DIGITAL MODEL IN THE CLOUD}

The next step is the digitization of all the information assets. This process represents the fundamental aspect on which the entire protocol is based both from a conceptual and operational point of view: building and hierarchizing in an appropriate way our "data container" is undoubtedly the necessary condition to implement advanced methodologies aimed at managing dynamic and interactive data.

Parallel to the creation of an IT archive of the property, a digital twin is produced, by means of computerized modelling B.I.M. able to combine technical and documentary contents with the geometric and performance data of the individual construction elements that are part of the building. In this way, the consultation of any information relating to the property in question is standardized and immediate; in the same way, it is possible to catalogue and use all the data implemented in a totally customized way, for example through the strengthening of a general structure of work division, created according to the needs of the case

The result to which the previous phases have led can be summarized as the creation of a database that is consistent, complete and updated with the current state of the property in question. The natural development of what has been prepared is the configuration of methods and tools for using the data itself; this allows, on an operational level, to program customized solutions in relation to the customer's needs, which can be combined, if necessary, with existing standards and procedures.

These data, therefore, are integrated and put in a one-to-one relationship within a space in the cloud that acts as a data store, designed to communicate with suitably configured IT tools and processes. The digital structure thus created allows you to connect, within the platform, the information related to ordinary and extra-ordinary maintenance processes to the elements making up the BIM., based on the level of detail required. This leads to enriching the static information modelling with dynamic data. In fact, within these procedures, management workflows are implemented that take into account the interaction of the various stakeholders (maintenance technician, building manager, expense manager,), who have access to permissions and customized methods of use depending on the role that they have.

The platform, thus populated by computer links between the current state of the property (static data) and the related management procedures (dynamic data), allows the analysis of 


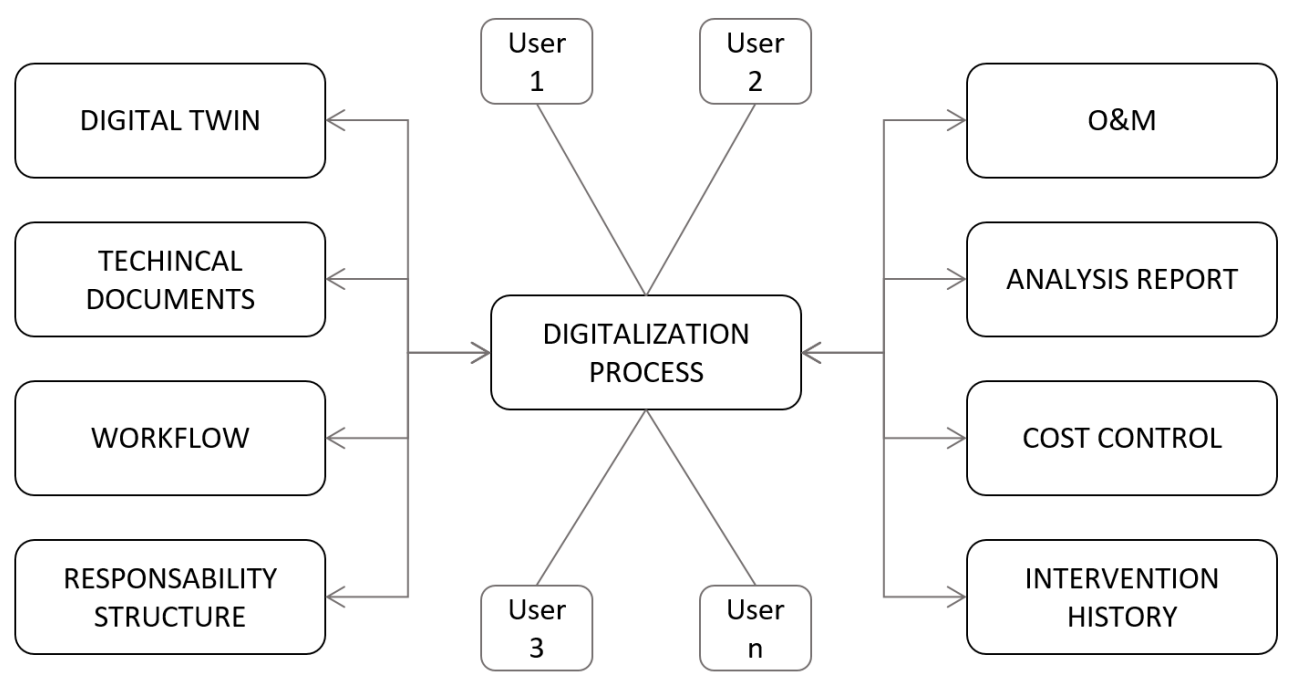

Figure 2: Functional diagram: Cloud platform.

the information collected through the creation of customized dashboards. This tool therefore aims to increase, in terms of transparency and process control, consultation and action by stakeholders. This therefore configures a common workspace capable of encouraging interaction and the definition of responsibilities, thus preventing inconsistencies and therefore costs and complications related to the ex-post resolution of problems.

The development of the IT structure described has led to the highlighting of four main areas, strongly correlated with each other, where direct or indirect advantages were found in the use of the digital tools associated with them:

- Reduction in the number of overall emergency interventions: the digital programming of ordinary maintenance procedures with automatic remind/alert systems has led to a stricter compliance with those which are the interventions foreseen on each component. It was highlighted how to punctually fulfill the deadlines set by the maintenance plan, an activity that is often complex to organize and carry out, is able by its nature to prevent some unexpected failure situations.

- Increase in the effectiveness of the operations: the analysis of the technical-construction nature of the property in question together with the subsequent continuous updates and additions with the platform containing the digital model, led to the configuration of a new unique and complete consultation tool. This prevents waste of time and economic resources, in light of possible quantitative and/or qualitative errors that may occur during the ordinary and extra-ordinary maintenance phases (for example where the need to replace a component occurs).

- Speed of processes: accessibility to the large amount of IT data in the cloud, necessary for carrying out maintenance procedures, has been made enormously faster and more direct, avoiding situations of inconvenience due to the unavailability of documentation or non-uniqueness of the data. Similarly, the communication systems developed have led to a direct information exchange structure, consistent with the procedures set out above and within them. 
- Control and transparency: the use of tools shared by the various stakeholders, within which it is possible to find all the information of the management procedures, has made it possible to carry out the tasks assigned to each of the parties in a more defined and clear way. The digital definition of responsibilities on a temporal and quantitative scale has also led to a significant increase in the transparency of the procedures in relation to the contractual obligations between the parties. The automatic generation of historical data relating to ordinary and extra-ordinary interventions have allowed a dynamic and constantly updated control both on the costs of the processes and on any anomalies, which can thus be detected and corrected in the shortest possible time.

From an economic point of view, in the face of a greater initial investment and longer initial times for the definition and development of the IT structure, a reduction in the costs of maintenance activities is obtained, as well as an increase in the quality of the service itself, compared to a "traditional" process (not integrated and/or digitized).

\section{APPLICATION TO THE CHAMBER OF COMMERCE BUILDING}

The case study concerns the digitization process of the Hadrianeum in Rome, one of the most representative buildings in the city and headquarter of the Rome Chamber of Commerce. The activities were divided into three main phases: digital survey and technological census, BIM digitalization, integration of FM processes within a cloud platform capable of connecting dynamic data to the various elements of the digital model. The result is a unique wired container customized to the needs of the various operators who daily enrich the digital database by carrying out their duties. Business intelligence tools have been integrated into the dynamism of the system to provide the property with a structured control over the costs and activities of FM, as a basis for making "well-informed-decisions".

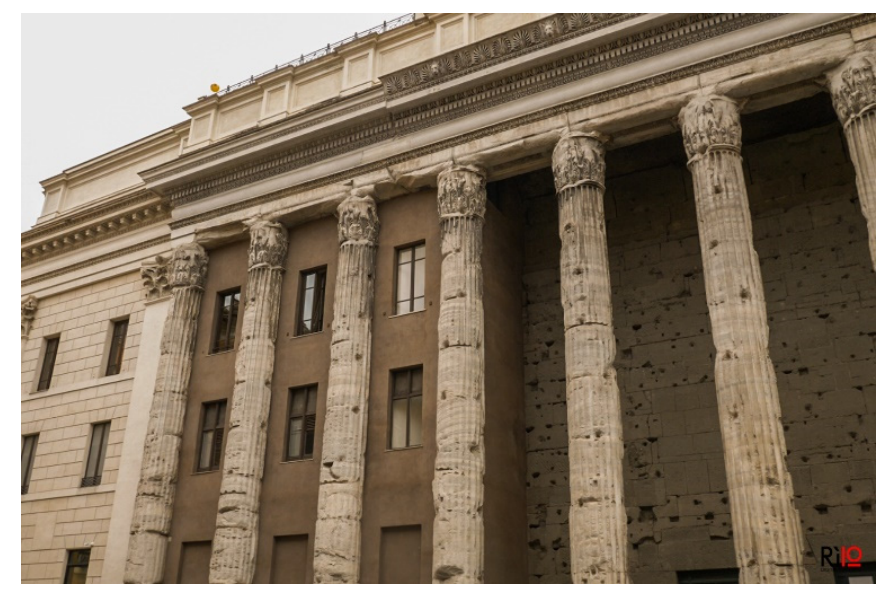

Figure 3: Exterior view: Hadrianeum.

The enormous construction and system complexity of the building was managed thanks to the use of innovative technologies, such as mobile laser scanning, which made it possible to carry out activities without interfering with the normal management of the Institution.

The process thus managed to trace 108 autonomous plants, 12 centralized systems, linked by $4.2 \mathrm{~km}$ of pipes. The transition from traditional practices to digital tools connected to the 
network and accessible remotely has also allowed a reduction in the carbon footprint of FM's activities, leading to a 100\% reduction in the paper used (about 22,000 sheets per year) and a waste reduction of material thanks to the simple and rapid availability of the technical information of each component.

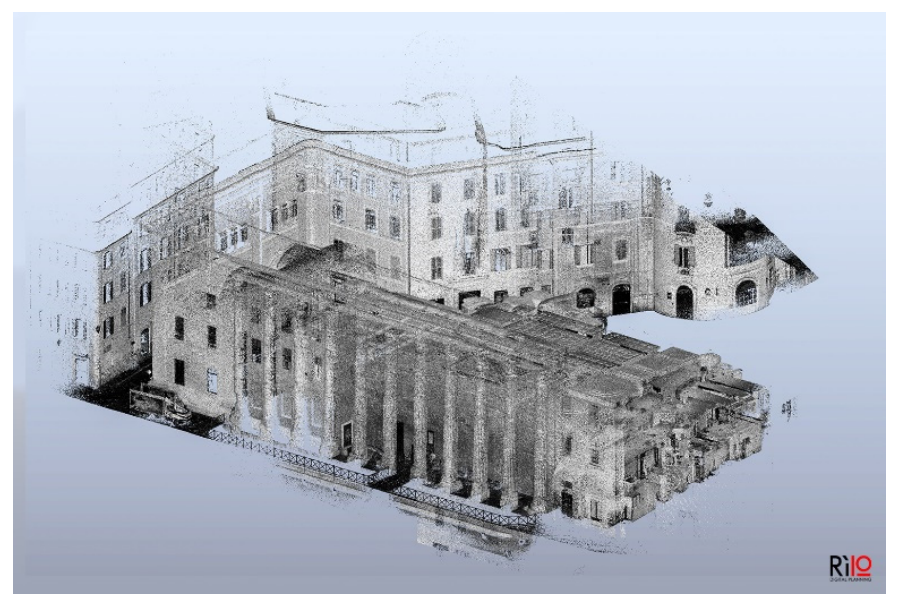

Figure 4: Point cloud model.

\section{CONCLUSION}

The case study, conducted on a historic building with "tertiary" use, made it possible to obtain the expected results. The objectives of the work, such as the recognition of the fault, the organized management of maintenance, the clear identification of all systems and components in the building, have been fully achieved. It also emerged that this protocol is more useful when the building has plant complexity. Further experiments with different buildings are underway, in this way it will be possible to evaluate the potential and reliability of the outputs in different contexts.

\section{REFERENCES}

[1] Di Ciaccio, D., Rossini, F.L. \& Maroder, E., From sheets to models: Innovative approaches for the facility and maintenance management. 3D Modeling \& BIM Data Modeling \& Management for AECO Industry, pp. 287-299, 2020.

[2] Bortoluzzi, B., Efremov, I., Medina, C., Sobieraj, D. \& McArthur, J.J., Automating the creation of building information models for existing buildings. Automation in Construction, 105, Elsevier: The Netherlands, 2019.

[3] Rossini, F.L., Fioravanti, A., Novembri, G. \& Insola, C., Holobuild: Process optimization by the introduction of mixed reality in construction site. Back to 4.0: Rethinking the Digital Construction Industry, eds. Ciribini et al., Maggioli Editore, pp. 279-288, 2016. 